\title{
Análise Psicométrica Preliminar de um Instrumento de Autorrelato para Avaliar Traços de Psicopatia
}

\author{
Nelson Hauck Filho - Universidade São Francisco, Itatiba, Brasil \\ Roberta Salvador-Silva - Universidade Federal do Rio Grande do Sul, Porto Alegre, Brasil \\ Marco Antônio Pereira Teixeira - Universidade Federal do Rio Grande do Sul, Porto Alegre, Brasil
}

\begin{abstract}
Resumo
Pesquisadores brasileiros dispunham, até o momento, de limitados recursos metodológicos para avaliar traços de psicopatia em indivíduos da população geral. O objetivo do presente estudo foi investigar as propriedades psicométricas de um novo instrumento de autorrelato de traços de psicopatia para ser usado em pesquisas com amostras da população geral brasileira. Os participantes do presente estudo foram 1238 indivíduos da população geral (idades de 18 a 76 anos; $M=28,36 ; D P=10,50$; $71,8 \%$ mulheres) que responderam a um questionário on-line. Métodos de Teoria de Resposta ao Item e análise fatorial ordinal ajudaram a reter um conjunto de 60 itens informativos das dimensões Egocentrismo Maquiavélico, Descontrole e Audácia. Escores latentes nesses domínios apresentaram, também, padrões de correlação teoricamente esperados com variáveis relevantes, incluindo uma avaliação independente de psicopatia - a escala Levenson Self-Report Psychopathy. Embora novos estudos psicométricos sejam necessários, as análises preliminares do instrumento revelaram adequadas propriedades psicométricas. Palavras-chave: Psicopatia, personalidade, Teoria de Resposta ao Item, análise fatorial.
\end{abstract}

\section{Preliminary Psychometric Analysis of a Self-Report Instrument for Assessing Psychopathic Traits}

\begin{abstract}
Brazilian researchers have dealt with limited methodological resources for assessing psychopathic traits of individuals from non-forensic settings. In the present study, we investigate the psychometric properties of a new self-report instrument for assessing psychopathic traits of individuals from the Brazilian general population. Participants were 1238 adults from Brazilian general population (ages from 18 to 76 years; $M=28.36 ; S D=10.50 ; 71.8 \%$ females), who answered an online questionnaire. We used exploratory ordinal factor analyses coupled with Item Response Theory (IRT) techniques to retain 60 highly informative items concerning the phenotypic dimensions of Machiavellian Egocentricity, Disinhibition and Fearless Dominance. Latent estimates of these three dimensions correlated with several theoretically relevant variables, including an external psychopathy assessment - the Levenson Self-Report Psychopathy scale. Despite the need of further studies, our findings suggest the new tool has adequate psychometric properties.

Keywords: Psychopathy, personality, Item Response Theory, factor analysis.
\end{abstract}

Análisis Psicométrico Preliminar de un Instrumento de Auto Relato para la Evaluación de Rasgos de Psicopatía

\begin{abstract}
Resumen
Investigadores brasileños disponían, hasta el momento, de herramientas metodológicas limitadas para evaluar rasgos psicopáticos en la población general. El objetivo de este estudio fue investigar las propiedades psicométricas de un nuevo instrumento de auto-reporte de rasgos psicopáticos para estudios con individuos de la población brasileña. Los participantes fueron 1.238 individuos (edades 18 a 76 años, $M=28.36, S D=10.50 ; 71.8 \%$ mujeres) que respondieron a un cuestionario online. Métodos robustos de análisis de datos categóricos ordinales ayudaron a mantener un conjunto de 60 ítems informativos de las dimensiones Egocentrismo Maquiavélico, Descontrol y Audacia. Las puntuaciones latentes en estas escalas también mostraron patrones de correlación esperados con variables teóricamente relevantes, incluyendo una evaluación independiente de psicopatía - la escala Levenson Self Report Psychopathy. Aunque se necesitan nuevos estudios psicométricos, el análisis preliminar del instrumento mostró propiedades psicométricas adecuadas.

Palabras clave: Psicopatía, personalidad, Teoría de Respuesta al Ítem, análisis factorial.
\end{abstract}

\section{Introdução}

"Personalidade psicopática" é um termo usado para se referir a um conjunto de características socialmente indesejáveis, manifestas nas esferas afetiva, interpessoal e comportamental (Cleckley, 1976; Cooke, Kosson, \& Michie, 2001). As principais dimensões da psicopatia são Meanness (isto é, falta de empatia, falta de remorso, manipulação), Disinhibition (isto é, impulsividade, falta de controle, uso de substâncias) e Boldness (isto é, dominância social, resiliência, baixo medo; Patrick, Fowles, \& Krueger, 2009; Skeem, Polaschek, Patrick, \& Lilienfeld, 2011). Indivíduos com níveis elevados de psicopatia tendem a ser pouco empáticos, pouco dispostos a colaborar com outras pessoas, e mais propensos ase comportar de forma agressiva e antissocial (Fowles \& Dindo, 2006). Essas características, combinadas com uma aparência de ajustamento 
psicossocial, resultam em uma configuração de personalidade de difícil manejo clínico e institucional (Edens, Poythress, Lilienfeld, Patrick, \& Test, 2008).

De um ponto de vista neuropsicológico, a característica principal da psicopatia é uma reduzida responsividade a estímulos aversivos, que se manifesta como dificuldade em experienciar medo e ansiedade (Corr, 2010; Hughes, Moore, Morris, \& Corr, 2012). Medo (um estado interno experienciado pelo organismo ao se afastar de uma fonte de perigo) e ansiedade (experienciada ao se aproximar, com cautela, de estímulos novos, aversivos ou mesmo potencialmente aversivos) possuem uma base neurobiológica com algumas distinções importantes. O medo é mediado por um sistema neuropsicológico conhecido como Fight-Flight-Freeze System (FFFS), enquanto a ansiedade é mediada pelo Behavioral Inbibition System (BIS) (Corr, 2010). Enquanto o FFFS evoluiu para proporcionar aos organismos escapar de perigos, o BIS possibilita distribuir melhor os recursos atencionais e resolver conflitos de metas - por exemplo, evitação versus aproximação (Corr \& McNaughton, 2008). Déficits no funcionamento de ambos os processos podem ter consequências que incluem o prejuízo quanto a antecipar ou responder a uma situação aversiva (Dindo \& Fowles, 2011; Patrick et al., 1993), dificuldades na formação de associações mediante condicionamento clássico aversivo (Flor, Birbaumer, Hermann, Ziegler, \& Patrick, 2002) e prejuízos na capacidade de reconhecer determinadas emoções negativas em outras pessoas (Wilson, Juodis, \& Porter, 2011). Essas características, se acompanhadas de um reduzido autocontrole e de uma elevada reatividade a estímulos que sinalizam recompensas (Corr, 2010), podem constituir um funcionamento psicológico de difícil socialização e propenso a problemas interpessoais de diversos níveis de severidade.

No cenário nacional, existem versões disponíveis em português brasileiro de alguns instrumentos que servem à avaliação de traços de psicopatia. Para o uso em contextos clínicos, forenses e prisionais, há adaptações da Psychopathy Checklist-Revised (PCL-R; Hare, 2003; adaptada por Morana, 2004), da Psychopathy Checklist: Youth Version (PCL: YV; Forth, Kosson, \& Hare, 1995; adaptada por Ronchetti, Davoglio, Salvador-Silva, Vasconcellos, \& Gauer, 2010) e da Interpersonal Measure of Psychopathy (IM-P; Kosson, Steuerwald, Forth, \& Kirkhart, 1997; adaptada por Davoglio, Gauer, Vasconcellos, \& Lühring, 2011). No entanto, para pesquisas com amostras de médio e grande porte com adultos da população geral, há apenas a escala Levenson Self-Report
Psychopathy (LSRP; Levenson, Kiehl, \& Fitzpatrick, 1995; adaptada por Hauck-Filho \& Teixeira, 2014), um instrumento de autorrelato relativamente breve $(26$ itens). Apesar de amplamente utilizada, a escala LSRP é limitada no sentido de que não oferece uma avaliação compreensiva dos diversos traços de psicopatia, como o faz o principal instrumento de autorrelato na área, o Psychopathic Personality Inventory (PPI; Lilienfeld \& Andrews, 1996). Em contraste, o PPI é um instrumento comercializado, de modo que sua adaptação não contribuiria no sentido de proporcionar uma ferramenta gratuita e de livre acesso a pesquisadores brasileiros.

Um novo instrumento compreensivo e de autorrelato poderia preencher uma importante lacuna no país. Particularmente, a ferramenta poderia potencializar o desenvolvimento de estudos na área da psicopatia em contextos não forenses e não prisionais, tópico ainda pouco explorado no cenário nacional. Seria útil que um instrumento dessa natureza fosse informativo não apenas das características afetivas, interpessoais e comportamentais tradicionalmente enfatizadas pela literatura (Cleckley, 1976; Cooke \& Michie, 2001), mas, também, dos aspectos relacionados ao funcionamento deficitário dos sistemas BIS e FFFS, como discutido anteriormente. Além de proporcionar informação útil de um ponto de vista clínico, esse novo recurso poderia facilitar a identificação de correlatos neurobiológicos em estudos experimentais subsequentes.

Tendo em vista essa necessidade, o objetivo do presente estudo foi investigar as propriedades psicométricas de um novo instrumento de autorrelato de traços de psicopatia para uso em estudos com amostras oriundas da população geral brasileira. Para a escolha dos itens dessa nova ferramenta, foram empregados diversos métodos estatísticos modernos adequados para dados categóricos ordinais. Esses métodos robustos possibilitaram a seleção de itens para cobrir diferentes regiões do traço latente. $\mathrm{O}$ instrumento foi também correlacionado às escalas BIS/BAS (Carver \& White, 1994), à escala LSRP et al., 1995) e à escala Zanon de Afetos Positivos e Negativos (Zanon, Bastianello, Pacico, \& Hutz, 2013). A relação dos fatores do instrumento com o autorrelato de envolvimento prévio em infrações legais foi também testada. Coerente com a literatura, as hipóteses foram de que os fatores do instrumento construído apresentariam correlações negativas com BIS, e positivas com BAS, com as subescalas do instrumento LSRP e com o autorrelato de infrações legais (Brinkley, Schmitt, Smith, \& Newman, 2001; Corr, 2010; Hughes et al., 2012). As variáveis 
de afetos positivos e negativos, por sua vez, foram coletadas para fins exploratórios, de modo a permitir entender melhor o funcionamento emocional relacionado a cada fator do instrumento elaborado.

\section{Método}

\section{Participantes}

Os participantes do presente estudo foram 1238 indivíduos da população geral (idades de 18 a 76 anos; $M=28,36 ; D P=10,50 ; 71,8 \%$ mulheres) que responderam a um questionário on-line. Os participantes eram residentes em 19 estados brasileiros, sendo 63,5\% do Rio Grande do Sul e 17,1\% de São Paulo (47\% estudantes universitários). Quanto à origem étnica, 86,99\% relataram ser brancos; $8,11 \%$ pardos; 3,3\% negros; $1,43 \%$ amarelos e $0,18 \%$ indígenas. A renda familiar mensal modal foi a categoria "acima de $\mathrm{R} \$ 7.000$ " $(22,73 \%)$, seguida de "entre $\mathrm{R} \$ 2.501$ e $\mathrm{R} \$ 3.000$ " (9,89\%) e “entre $\mathrm{R} \$ 2.001$ e $\mathrm{R} \$ 2.500 ”(9 \%)$. Com relação ao status de relacionamento, $37,52 \%$ declararam-se solteiros; $34,22 \%$ namorando; $24,06 \%$ casados; 3,39\% divorciados e $0,8 \%$ viúvos. Dentre esses indivíduos, $4,1 \%$ afirmaram já ter tido contato com o sistema judicial em função de flagrante delito ou queixa prestada por terceiros, sendo 20,76 anos a média de idade para a primeira vez em que isso ocorreu $(D P=7,86)$.

\section{Instrumentos}

\section{Itens de psicopatia}

De modo análogo ao estudo de Lilienfeld e Andrews (1996) de construção do Psychopathic Personality Inventory, a redação dos itens iniciou com a definição de construtos focais. No presente caso, o ponto de partida foi uma revisão sistemática e análise qualitativa sobre a estrutura fatorial de 38 instrumentos de avaliação de traços de psicopatia. Os autores identificaram nove aspectos distintos enfatizados pela literatura psicométrica tradicional: tendências antissociais, baixo autocontrole, dependência de recompensas, cinismo, exploração interpessoal, frieza emocional, narcisismo patológico, dominância social e baixa reatividade aversiva. Como a categoria de baixa reatividade aversiva consistiu em uma mistura de aspectos de medo e ansiedade, optou-se por dividi-la em: despreocupação (baixo BIS, baixa ansiedade; Corr, 2010) e intrepidez (baixo FFFS, reduzido medo; Corr, 2010). Disso, resultaram 10 construtos focais a partir dos quais foram elaborados os itens do instrumento, de modo a buscar proporcionar uma avaliação compreensiva dos principais traços de psicopatia.

Os itens foram escritos de modo a maximizar a cobertura do traço latente e evitar a sua redundância semântica. Além de representativos dos 10 construtos focais, os itens foram escritos para que ficassem dispostos em um contínuo de psicopatia, abrangendo desde níveis baixos a níveis muito altos, ou seja, a intenção foi promover uma variabilidade no parâmetro da dificuldade dos itens. Sete juízes com experiência na área da psicopatia ou da construção de instrumentos avaliaram a representatividade dos construtos focais e a clareza de escrita dos itens. Além disso, foram conduzidas três entrevistas com indivíduos com baixa escolaridade (até ensino fundamental completo), de modo a certificar que as sentenças e a instrução de resposta contivessem palavras acessíveis, também, ao estrato educacional inferior da população. Finalmente, uma versão preliminar dos itens foi aplicada a uma amostra-piloto de 224 estudantes de graduação de cursos da Universidade Federal do Rio Grande do Sul (58,9\% do sexo feminino, idades entre 18 e 54 anos, média $=23,76, D P=$ $8,02)$. Análises exploratórias dos dados orientaram a escrita de novos itens e a reformulação de outros. Após todas essas etapas, um conjunto final de 84 itens de autorrelato foi considerado adequado para as coletas. A escala de resposta foi definida como $1=$ definitivamente não, $2=$ acho que não, $3=$ acho que sim e $4=$ definitivamente sim.

\section{Escalas Behavioral Inhibition / Behavioral Activation (BIS/ BAS; Carver \& White, 1994)}

As escalas BIS/BAS foram desenvolvidas para avaliar os sistemas neuropsicológicos BIS e BAS (ver Corr, 2008). Para o presente estudo, foi preparada uma versão em português brasileiro das escalas BIS/BAS, seguindo uma sequência de 1) tradução pelo grupo de pesquisa, levando em consideração os aspectos teóricos avaliados, 2) análise das traduções por dois juízes bilíngues e 3) ajustes finais nas traduções. O instrumento é composto por 20 itens avaliados em escala Likert $(1=$ discordo totalmente e $5=$ concordo totalmente) distribuídos em quatro escalas: BAS-Drive(BAS-D), BAS-Reward Responsiveness (BAS-RR), BAS-Fun seeking (BAS-F) e BIS. No presente estudo, uma análise fatorial confirmatória categóricaWeighted Least Squares Mean-and Variance-adjusted (WLSMV; Muthén, Du Toit, \& Spisic, 1997), revelou um ajuste bastante ruim para a estrutura original do instrumento, $\chi^{2}=1306,67, g l=164, p<0,001$, RMSEA $=$ 0,087 (CI 90\% 0,083-0,092), CFI = 0,687, TLI =0,638. 
A consistência interna ( $\alpha$ de Cronbach) foi 0,56 para BAS-D; 0,54 para BAS-RR; 0,58 para BAS-F e 0,57 para BIS. Apesar falta de ajuste e da reduzida consistência interna, o instrumento foi aplicado por ser um dos correlatos mais frequentes de instrumentos de psicopatia (Newman \& Malterer, 2009).

\section{Escala de Afetos Zanon (EAZ; Zanon et al., 2013)}

O instrumento avalia disposições dos indivíduos à experiência de afetos positivos e negativos. $\mathrm{O}$ seu papel, no presente estudo, foi investigar a validade discriminante das dimensões do instrumento elaborado, dadas as relações específicas de traços de psicopatia com afetos positivos e negativos (Patrick et al., 2009). Foram aplicados os 20 itens da escala, avaliados em uma escala Likert de cinco pontos, $1=$ discordo totalmente e $5=$ concordo totalmente. $\mathrm{Na}$ presente amostra, a consistência interna ( $\alpha$ de Cronbach) foi de 0,83 para a escala de afetos positivos e 0,86 para a escala de afetos negativos.

\section{Autorrelato de Infrações Legais}

O autorrelato de infrações legais foi avaliado por meio de questão única, na qual os participantes eram solicitados a indicar se, alguma vez na vida, haviam sido acusados formalmente de terem cometido um ato infracional de qualquer tipo. Na sequência, os participantes também foram solicitados a indicar o número de vezes em que isso ocorreu; essa segunda variável não foi empregada no presente estudo por gerar os mesmos resultados (na análise de regressão empregada - ver adiante) que a simples resposta dicotômica à primeira questão.

\section{Escala Levenson Self-Report Psychopathy Scale (LSRP;}

Levenson et al., 1995)

Foi empregada a versão adaptada ao Brasil por Hauck-Filho e Teixeira (2014). A escala LSRP é um dos instrumentos de autorrelato mais utilizados para avaliar psicopatia (Lilienfeld \& Fowler, 2006), sendo incluído nas coletas para investigar a validade convergente e discriminante (das escalas) do instrumento desenvolvido no presente estudo. O instrumento é composto por 26 itens de autorrelato avaliados em escala Likert $(1=$ discordo totalmente e $5=$ concordo totalmente), distribuídos em duas dimensões: Psicopatia Primária e Psicopatia Secundária. No presente estudo, a consistência interna ( $\alpha$ de Cronbach) foi 0,83 para Psicopatia Primária, 0,68 para Psicopatia Secundária e 0,82 para o total de itens.

\section{Procedimentos}

As coletas foram feitas on-line por meio da plataforma Survey Monkey. Os participantes foram contatados via e-mail, redes sociais e pessoalmente, na instituição de origem dos autores do estudo. Os indivíduos acessaram um endereço eletrônico que esteve disponível entre o período de maio de 2012 e abril de 2013. Há evidências de uma adequada equivalência entre dados coletados presencialmente, com o tradicional método lápis-papel, e coletados on-line (Brock, Barry, Lawrence, Dey, \& Rolffs, 2012). Tanto os participantes dessa coleta quanto aqueles do estudo-piloto assinaram termos de consentimento informado antes de responder aos instrumentos.

A análise psicométrica do instrumento se baseou em uma combinação de métodos de análise fatorial exploratória categórica/Teoria de Resposta ao Item (TRI), tendo em vista a natureza dos dados categóricos ordinais da escala Likert utilizada. A dimensionalidade dos itens foi investigada mediante o método Minimum Average Partial (MAP; Velicer, 1976), que aponta o número de fatores que resulta nas menores correlações parciais residuais entre os itens. O método de estimação da análise fatorial foi Unweighted Least Squares (ULS), em conjunto com rotação oblíqua promin, baseados em uma matriz de correlações policóricas. As análises foram conduzidas com o programa Factor 9.2 (Lorenzo-Seva \& Ferrando, 2006).

Os itens de cada fator foram posteriormente avaliados com dois modelos de TRI. O primeiro deles foi o Rating Scale Model (RSM; Andrich, 1978). O RSM assume que as curvas características dos itens são paralelas (Embretson \& Reise, 2000). O ajuste dos itens é avaliado a partir dos índices infit e outfit, que devem estar entre 0,60 e 1,40 (Linacre, 2011). No caso da escala Likert de quatro pontos do instrumento, o RSM estima os três pontos de corte ou thresholds que indicam o lugar, no traço latente, em que há igual probabilidade de endossar as categorias um e dois $\left(\tau_{1}\right)$, dois e três $\left(\tau_{2}\right)$, e três e quatro $\left(\tau_{3}\right)$. As distâncias entre os parâmetros $\tau$ são estimadas como fixas para todos os itens, variando entre os itens a localização $(\delta$ ) da estrutura fixa no contínuo latente - um parâmetro geral de dificuldade do item.

O segundo modelo utilizado foi o Graded Response Model (GRM; Samejima, 1969). Um pouco diferente do RSM, no GRM, os thresholds (dificuldades) $b_{1}, b_{2}$, e $b_{3}$ representam o ponto em que há $50 \%$ de probabilidade de responder acima de um, dois e três na escala, respectivamente. Outro parâmetro estimado é o $a$, que 
é análogo à carga fatorial e indica a discriminação do item. As curvas de informação dos fatores, geradas a partir do GRM, também, foram avaliadas. Curvas de informação ilustram quais as regiões do traço latente são avaliadas com maior precisão pelos itens do instrumento, constituindo uma análise em profundidade da precisão do instrumento para cada nível específico do traço latente (Embretson \& Reise, 2000). Foram utilizados os programas Winsteps 3.72 (Linacre, 2011) e o pacote ltm para o programa R (Rizopoulos, 2006). Em todas as análises, foi fixada em 0,00 a média do nível de traço latente dos indivíduos para fins de identificação do modelo. Para as análises subsequentes, foram empregados os escores fatoriais obtidos para o modelo final.

\section{Resultados}

\section{Dimensionalidade}

Os dados se mostraram adequados para a análise fatorial exploratória ordinal ULS baseada em correlações policóricas, $\mathrm{KMO}=0,90, \chi^{2}$ de Bartlett $=18750,9$, $p<0,001$. O método MAP sugeriu a retenção de três fatores explicativos de $28,62 \%$ da variância total dos itens, o que manteria as correlações residuais parciais a uma média de apenas 0,005 .

Especificando-se um número fixo de três fatores, 20 itens apresentaram cargas fatoriais abaixo de 0,30 nos três fatores e quatro itens se mostraram incoerentes com as dimensões teóricas esperadas, sendo então excluídos para uma nova análise. A nova análise exploratória com 60 itens resultou em um índice de ajuste Global Fit Index (GFI) = 0,96, sendo explicados $33,71 \%$ da variância total dos itens [autovalores $=12,38(20,64 \%) ; 4,47(7,44 \%)$ e $3,37(5,62 \%)]$. O primeiro fator explicou os aspectos de baixo autocontrole, impulsividade, dominância e comportamentos desviantes, sendo denominado "Descontrole." O segundo fator foi explicativo de características de manipulação, frieza emocional, cinismo e narcisismo patológico, sendo denominado "Egocentrismo Maquiavélico". Por sua vez, o terceiro fator explicou os aspectos de baixo BIS (reduzida ansiedade) e baixo FFFS (reduzido medo), sendo chamado de "Audácia". A distribuição dos itens nos fatores é apresentada na Tabela 1. A correlação entre Descontrole e Egocentrismo Maquiavélico foi de 0,63, ao passo que as correlações entre Audácia e Egocentrismo Maquiavélico, e Audácia e Descontrole foram, respectivamente, $-0,10$ e $-0,14$.

\section{Análises de TRI}

$\mathrm{Na}$ Tabela 2, são apresentados os resultados das análises de TRI dos itens do fator Descontrole. Apenas os itens 01 e 42 estiveram ligeiramente fora do intervalo de valores entre 0,60 e 1,40 para os índices infit e outfit do RSM. Ambos foram mantidos, em função de os desvios serem de baixa magnitude. Os itens estão apresentados ordenados em função do parâmetro $\delta_{i}$ - quanto maior o valor do parâmetro, mais severa é a característica avaliada pelo item. Ou seja, sentir tédio facilmente foi o aspecto menos severo (item 02) e ameaçar outras pessoas foi o aspecto mais severo da escala (item 38). Os parâmetros $a$ do GRM, a seu turno, sugeriram uma boa capacidade discriminativa para todos os itens (valores acima de 0,50).

$\mathrm{Na}$ sequência, as mesmas análises foram feitas com os itens do fator Egocentrismo Maquiavélico. $\mathrm{Na}$ Tabela 3, pode-se ver como apenas os itens 04,37 e 46 estiveram ligeiramente fora do intervalo de valores de 0,60-1,40 para os índices infit e outfit. Em função de os desvios serem pequenos, esses itens foram mantidos, resultando em bons níveis de fidedignidade para os itens e para as pessoas. Todos os itens, exceto 04 e 29 , apresentaram uma discriminação empírica acima de 0,50 .

Os 13 itens do fator Audácia apresentaram um excelente ajuste ao RSM, de acordo com os índices infit e outfit, bem como um valor máximo em termos de fidedignidade (Tabela 4). Apesar de a cobertura do traço latente ter sido um pouco mais modesta do que os itens de Descontrole e Egocentrismo Maquiavélico, a escala Audácia foi, ainda assim, abrangente de um amplo espectro do traço latente, como visto pelos parâmetros $\delta, b$ e. Observaram-se também valores aceitáveis para os parâmetros $a$ da discriminação.

Por fim, foram investigadas as curvas de informação de cada uma das três escalas. O total de pontos de informação foi: Descontrole $=45,38$; Egocentrismo Maquiavélico $=46,47$; e Audácia $=23,49$. A representação gráfica das curvas para o intervalo de -4,0 e 4,0 logits é apresentada na Figura 1. Houve uma tendência de os itens das três escalas serem mais informativos para a porção superior do traço latente, sendo a escala de Egocentrismo Maquiavélico a mais discriminativa dentre as três.

\section{Evidências de Validade Convergente para as Escalas}

Os resultados das análises correlacionais entre os fatores e as demais variáveis são mostrados na Tabela 5 . Observa-se que os fatores Descontrole e Egocentrismo 
Tabela 1

Análise Fatorial Exploratória Unweighted Least Squares com Correlações Policóricas

\begin{tabular}{|c|c|c|c|c|}
\hline Item & F 1 & $\mathrm{~F} 2$ & F 3 & $H^{2}$ \\
\hline 26. Considera-se uma pessoa rebelde? & 0,73 & $-0,24$ & 0,06 & 0,36 \\
\hline $\begin{array}{l}\text { 18. Costuma se aproximar de algo que pode ser perigoso, mesmo quando vê os } \\
\text { outros se afastando? }\end{array}$ & 0,63 & $-0,01$ & 0,28 & 0,42 \\
\hline 11. Gosta da sensação de fazer coisas que podem oferecer risco à sua vida? & 0,62 & $-0,04$ & 0,16 & 0,35 \\
\hline $\begin{array}{l}\text { 41. Gosta de fazer as suas próprias regras, sem se importar com aquilo que as } \\
\text { outras pessoas consideram certo ou errado? }\end{array}$ & 0,62 & $-0,06$ & 0,15 & 0,35 \\
\hline 12. É o tipo de pessoa que "não leva desaforos para casa"? & 0,61 & $-0,30$ & $-0,00$ & 0,24 \\
\hline $\begin{array}{l}\text { 52. Costuma ouvir os outros dizerem que você é uma pessoa muito teimosa ou } \\
\text { "cabeça-dura"? }\end{array}$ & 0,59 & $-0,24$ & $-0,13$ & 0,26 \\
\hline $\begin{array}{l}\text { 47. Gosta de intimidar outras pessoas encarando-as diretamente nos olhos sem } \\
\text { desviar o olhar? }\end{array}$ & 0,53 & 0,08 & $-0,00$ & 0,34 \\
\hline $\begin{array}{l}\text { 19. Em diversas situações, precisa se controlar para não fazer ou dizer coisas que } \\
\text { trarão problemas a você? }\end{array}$ & 0,52 & $-0,08$ & $-0,07$ & 0,24 \\
\hline $\begin{array}{l}\text { 35. Quebra coisas ou age agressivamente quando está com raiva por algo que } \\
\text { aconteceu a você? }\end{array}$ & 0,50 & 0,01 & $-0,15$ & 0,31 \\
\hline $\begin{array}{l}\text { 3. Costuma perder a paciência rapidamente quando precisa ficar esperando por } \\
\text { algo, como um ônibus que não vem? }\end{array}$ & 0,50 & $-0,19$ & $-0,31$ & 0,30 \\
\hline 10. Tem dificuldade para acatar ordens de chefes ou superiores? & 0,48 & $-0,00$ & 0,03 & 0,23 \\
\hline $\begin{array}{l}\text { 30. Tenta fazer a sua opinião prevalecer a todo custo quando está debatendo um } \\
\text { assunto com outras pessoas? }\end{array}$ & 0,47 & $-0,03$ & $-0,18$ & 0,25 \\
\hline $\begin{array}{l}\text { 42. Usaria drogas ilícitas (maconha, cocaína, etc.) apenas para conhecer como é a } \\
\text { sensação? }\end{array}$ & 0,46 & 0,02 & 0,07 & 0,22 \\
\hline $\begin{array}{l}\text { 24. Aceitaria um emprego que pagasse bem, mas que envolvesse risco de vida o } \\
\text { tempo todo? }\end{array}$ & 0,46 & 0,05 & 0,24 & 0,27 \\
\hline 6. Gosta que os outros tenham medo de você? & 0,42 & 0,32 & 0,05 & 0,44 \\
\hline 1. Quando era menor, costumava se envolver em brigas com as outras crianças? & 0,41 & $-0,11$ & 0,07 & 0,12 \\
\hline 2. Sente tédio facilmente se fica sem fazer alguma coisa? & 0,40 & $-0,14$ & $-0,19$ & 0,16 \\
\hline $\begin{array}{l}\text { 38. Às vezes, usa de ameaças (falsas ou verdadeiras) para convencer as pessoas a } \\
\text { fazerem o que você quer? }\end{array}$ & 0,38 & 0,24 & $-0,13$ & 0,35 \\
\hline $\begin{array}{l}\text { 43. Costuma ficar pensando sobre como se vingaria de pessoas que machucaram } \\
\text { ou magoaram você? }\end{array}$ & 0,36 & 0,34 & $-0,14$ & 0,43 \\
\hline $\begin{array}{l}\text { 15. Gosta quando tem a oportunidade de exercer a sua autoridade sobre outras } \\
\text { pessoas mandando-as fazerem coisas para você? }\end{array}$ & 0,34 & 0,30 & $-0,22$ & 0,41 \\
\hline $\begin{array}{l}\text { 7. Tenta, com frequência, fazer os outros acreditarem que você sabe mais do que } \\
\text { realmente sabe sobre um determinado assunto? }\end{array}$ & 0,31 & 0,29 & $-0,07$ & 0,30 \\
\hline $\begin{array}{l}\text { 44. Socorreria alguém que estivesse precisando de ajuda mesmo se soubesse que } \\
\text { ninguém ficaria sabendo da sua boa ação ou que você não receberia nada em } \\
\text { troca fazendo isso? (Reverso) }\end{array}$ & $-0,36$ & 0,77 & 0,09 & 0,38 \\
\hline $\begin{array}{l}\text { 37. Sente indignação ao ver uma pessoa decente e trabalhadora sofrer uma } \\
\text { injustiça? (Reverso) }\end{array}$ & $-0,23$ & 0,73 & 0,21 & 0,40 \\
\hline 54. Sente prazer em presenciar o sofrimento das outras pessoas? & 0,09 & 0,66 & 0,09 & 0,52 \\
\hline $\begin{array}{l}\text { 28. Deixaria de ganhar um bom dinheiro para poder salvar a vida de alguém que } \\
\text { convive com você? (Reverso) }\end{array}$ & $-0,22$ & 0,66 & 0,03 & 0,30 \\
\hline
\end{tabular}


Tabela 1

Análise Fatorial Exploratória Unweighted Least Squares com Correlações Policóricas (Continuação)

\begin{tabular}{lcccc}
\hline Item & F 1 & F 2 & F 3 & $H^{2}$ \\
\hline 22. Algumas vezes, não dá a mínima quando sabe que alguém está passando & $-0,03$ & $\mathbf{0 , 6 5}$ & 0,09 & 0,39
\end{tabular}

por um momento ruim, mesmo que essa pessoa seja um amigo ou familiar com quem você não esteja brigado?

53. Tenta agir com honestidade, mesmo quando percebe que os outros ao seu redor não agem da mesma maneira? (Reverso)

36. Trabalharia com pessoas inescrupulosas e sem ética se tivesse a chance de ganhar algum bom dinheiro em troca?

34. Pegaria para você algo valioso de outra pessoa se soubesse que ninguém ficaria sabendo?

45. Em um dia de bom humor, consegue se sentir indiferente, como se não tivesse nada a ver com a situação, ao ver um cachorro ou outro animal sendo maltratado?

48. Seria capaz de fingir que defende uma ideia ou crença religiosa se isso trouxesse dinheiro ou algum tipo de vantagem para você?

16. Algumas vezes, finge gostar de uma pessoa apenas para tirar vantagem da situação?

23. Considera o status social ou a riqueza das pessoas como um fator importante para decidir com quem se relacionar em termos de amizade ou namoro?

17. Tenta, em geral, passar uma imagem de bom cidadão, ainda que tenha que enganar as pessoas em certas ocasiões?

31. Quando conveniente, finge que possui mais necessidade do que as outras pessoas para obter vantagens, como atendimento prioritário ou atenção especial? 57. Mudaria completamente o seu jeito de agir para ser aceito por um grupo de pessoas?

29. Pensa mais nas necessidades dos outros do que nas próprias necessidades? (Reverso)

46. Sente prazer em humilhar pessoas que você julga serem inferiores?

56. Seria capaz de se comportar de maneira agressiva e hostil para com outras pessoas se isso fizesse você conseguir alguma coisa que quisesse muito?

14. Sente prazer em despertar inveja nas outras pessoas?

27. Conseguiria perdoar alguém que, sem querer, quebrasse alguma coisa sua da qual você gostava muito? (Reverso)

55. Gosta de receber privilégios, tratamento diferenciado ou parecer mais importante do que as outras pessoas em uma situação social?

20. Em comparação a seus amigos, tem mais preconceito do que eles quanto a aceitar pessoas com crenças ou estilo de vida muito diferentes dos seus?

4. Sente culpa depois que diz ou faz algo que pode ter magoado outra pessoa? (Reverso)

21. Considera ser melhor do que seus amigos ou amigas em inventar desculpas esfarrapadas para não ter que assumir a responsabilidade por algo que você fez?

$-0,09 \quad \mathbf{0 , 6 1} \quad-0,05 \quad 0,32$

$0,09 \quad \mathbf{0 , 5 8} \quad 0,05 \quad 0,41$

$0,08 \quad \mathbf{0 , 5 8} \quad 0,01 \quad 0,40$

$-0,17 \quad \mathbf{0 , 5 7} \quad 0,16 \quad 0,25$

$0,13 \quad \mathbf{0 , 5 7} \quad-0,08 \quad 0,45$

$0,14 \quad \mathbf{0 , 5 7} \quad-0,13 \quad 0,48$

$\begin{array}{llll}-0,15 & \mathbf{0 , 5 2} & -0,12 & 0,21\end{array}$

$0,18 \quad \mathbf{0 , 5 1} \quad-0,13 \quad 0,44$

$0,13 \quad \mathbf{0 , 4 7} \quad-0,16 \quad 0,36$

$0,01 \quad \mathbf{0 , 4 6} \quad-0,07 \quad 0,23$

$\begin{array}{llll}-0,22 & \mathbf{0 , 4 5} & 0,04 & 0,13\end{array}$

$0,23 \quad \mathbf{0 , 4 5} \quad 0,00 \quad 0,38$

$\mathbf{0 , 3 0} \quad \mathbf{0 , 4 5} \quad-0,01 \quad 0,47$

$0,23 \quad \mathbf{0 , 4 2} \quad-0,23 \quad 0,44$

$\begin{array}{llll}-0,05 & \mathbf{0 , 4 1} & -0,11 \quad 0,17\end{array}$

$0,19 \quad \mathbf{0 , 4 0} \quad-0,23 \quad 0,38$

$-0,11 \quad \mathbf{0 , 4 0} \quad-0,03 \quad 0,12$

$-0,09 \quad \mathbf{0 , 3 9} \quad \mathbf{0 , 3 1} \quad 0,19$

$0,28 \quad \mathbf{0 , 3 7} \quad 0,00 \quad 0,35$

5. Pensa, quando está em meio a colegas de trabalho ou estudo, que são todos inferiores quando comparados a você?

13. Tem pouco interesse pelas pessoas, a ponto de se questionar se já amou alguém de verdade?

$0,14 \quad \mathbf{0 , 3 6} \quad 0,04 \quad 0,21$

$0,22 \quad \mathbf{0 , 3 2} \quad 0,08 \quad 0,24$ 
Tabela 1

Análise Fatorial Exploratória Unweighted Least Squares com Correlações Policóricas (Continuação)

\begin{tabular}{|c|c|c|c|c|}
\hline Item & F 1 & F 2 & F 3 & $H^{2}$ \\
\hline $\begin{array}{l}\text { 50. Teria medo de acampar em uma floresta sem ninguém fazendo companhia a } \\
\text { você? (Reverso) }\end{array}$ & 0,26 & $-0,02$ & 0,71 & 0,52 \\
\hline 59. Teria receio de entrar, à noite, em uma casa abandonada? (Reverso) & 0,24 & 0,05 & 0,68 & 0,48 \\
\hline $\begin{array}{l}\text { 49. Sente ansiedade ou nervosismo quando sabe que precisa ter uma conversa } \\
\text { difícil com alguém? (Reverso) }\end{array}$ & $-0,15$ & 0,16 & 0,57 & 0,35 \\
\hline 60. Ficaria alerta se tivesse que andar em uma rua deserta à noite? (Reverso) & 0,18 & 0,14 & 0,49 & 0,28 \\
\hline $\begin{array}{l}\text { 33. Temeria subir } 20 \text { andares em um elevador com o chão e as paredes feitas de } \\
\text { vidro? (Reverso) }\end{array}$ & 0,17 & $-0,01$ & 0,45 & 0,20 \\
\hline $\begin{array}{l}\text { 39. Assusta-se quando está andando na calçada e, de repente, um cachorro late } \\
\text { para você por trás da cerca do pátio de uma casa? (Reverso) }\end{array}$ & 0,04 & 0,08 & 0,42 & 0,17 \\
\hline $\begin{array}{l}\text { 9. Costuma sentir medo a ponto de congelar e ficar sem reação em situações de } \\
\text { grande perigo? (Reverso) }\end{array}$ & 0,01 & 0,02 & 0,41 & 0,17 \\
\hline 25. Sente medo de contrair uma doença incurável? (Reverso) & $-0,08$ & 0,01 & 0,38 & 0,16 \\
\hline $\begin{array}{l}\text { 51. Sente irritação facilmente quando está em um ambiente muito barulhento? } \\
\text { (Reverso) }\end{array}$ & $-0,19$ & 0,03 & 0,37 & 0,18 \\
\hline $\begin{array}{l}\text { 40. É o tipo de pessoa que esquece o lado positivo das coisas quando algo de } \\
\text { errado acontece? (Reverso) }\end{array}$ & $-0,19$ & $-0,10$ & 0,36 & 0,23 \\
\hline $\begin{array}{l}\text { 58. Consegue não pensar a respeito se sabe que algo de ruim está prestes a } \\
\text { acontecer a você? }\end{array}$ & 0,04 & 0,19 & 0,36 & 0,16 \\
\hline $\begin{array}{l}\text { 8. Costuma ficar com as mãos suadas de ansiedade por causa de compromissos } \\
\text { importantes que precisa fazer? (Reverso) }\end{array}$ & $-0,17$ & 0,05 & 0,32 & 0,13 \\
\hline $\begin{array}{l}\text { 32. Falaria tranquilamente se tivesse que fazer um discurso sobre os seus piores } \\
\text { defeitos a uma plateia? (Reverso) }\end{array}$ & 0,28 & $-0,17$ & 0,31 & 0,13 \\
\hline Fidedignidade dos fatores rotacionados & 0,91 & 0,93 & 0,85 & - \\
\hline Alpha de Cronbach & 0,84 & 0,84 & 0,69 & - \\
\hline Lambda2 & 0,84 & 0,85 & 0,70 & \\
\hline
\end{tabular}

Maquiavélicos correlacionaram positivamente com aspectos como BAS (exceto BAS-RR) e afetos negativos, e negativamente com BIS e afetos positivos. Descontrole e Egocentrismo Maquiavélicos diferenciaram em suas correlações com a escala LSRP. Especificamente, escores no domínio Egocentrismo Maquiavélicos estiveram mais fortemente relacionados à psicopatia primária $(r=0,66)$ do que à psicopatia secundária $(r=$ $0,36)$, enquanto escores em Descontroles e se correlacionaram mais com os aspectos secundários $(r=0,61)$ do que com os traços primários da psicopatia $(r=0,46)$. Audácia, por sua vez, correlacionou-se negativamente com BAS, BIS e afetos negativos, e positivamente com afetos positivos; esteve fraca e negativamente correlacionada com aspectos da escala LSRP.

Por fim, um modelo de regressão logística foi usado para investigar se os fatores seriam capazes de predizer o autorrelato de envolvimento prévio com a lei. Os resultados mostraram significância estatística para o modelo de regressão, $\chi^{2}=20,10, g l=3, p<0,001$. Especificamente, o fator Descontrole se mostrou significativa e positivamente associado a um histórico de infrações legais, $B=0,82$, teste de Wald $=15,85, g l=$ $1, p<0,001$.

\section{Discussão}

As estratégias robustas de análise empregadas recomendaram manter, na versão final do instrumento, 60 dos 84 itens construídos. Foi satisfeito o pressuposto da independência local (dos itens) dos modelos de variáveis latentes empregados, já que cada item obteve carga fatorial de moderada a alta em um dos fatores Egocentrismo Maquiavélico, Audácia e Descontrole, 
Tabela 2

Análises de Teoria de Resposta ao Item da Escala Descontrole (21 itens)

\begin{tabular}{|c|c|c|c|c|c|c|c|}
\hline \multirow{2}{*}{ Item } & \multicolumn{3}{|c|}{$\mathrm{RSM}^{\mathrm{a}}$} & \multicolumn{4}{|c|}{ GRM } \\
\hline & Infit & Outfit & $\delta_{i}$ & $b_{1}$ & $b_{2}$ & $b_{3}$ & $a$ \\
\hline 38 & 1,03 & 0,97 & 1,64 & 0,92 & 2,60 & 4,62 & 1,30 \\
\hline 06 & 0,95 & 1,00 & 1,49 & 0,72 & 2,63 & 4,55 & 1,52 \\
\hline 11 & 1,05 & 0,94 & 1,30 & 0,39 & 2,02 & 3,68 & 1,14 \\
\hline 47 & 1,04 & 0,95 & 1,28 & 0,43 & 2,00 & 3,85 & 1,34 \\
\hline 01 & 1,33 & 1,48 & 1,21 & 0,29 & 1,69 & 2,74 & 0,58 \\
\hline 35 & 1,03 & 1,01 & 1,12 & 0,14 & 1,75 & 3,40 & 1,17 \\
\hline 10 & 0,89 & 0,92 & 1,12 & $-0,16$ & 1,94 & 3,70 & 0,96 \\
\hline 18 & 0,94 & 0,96 & 1,08 & $-0,04$ & 1,77 & 3,71 & 1,19 \\
\hline 24 & 1,05 & 1,11 & 1,04 & $-0,12$ & 1,62 & 3,28 & 0,88 \\
\hline 15 & 0,97 & 0,93 & 0,83 & $-0,39$ & 1,33 & 3,18 & 1,22 \\
\hline 26 & 0,78 & 0,79 & 0,80 & $-0,73$ & 1,54 & 3,59 & 1,17 \\
\hline 42 & 1,49 & 1,49 & 0,74 & 0,09 & 0,73 & 2,06 & 0,84 \\
\hline 07 & 0,87 & 0,88 & 0,70 & $-0,82$ & 1,22 & 3,27 & 1,10 \\
\hline 41 & 0,96 & 1,00 & 0,60 & $-0,75$ & 1,14 & 2,61 & 1,17 \\
\hline 43 & 1,09 & 1,06 & 0,60 & $-0,45$ & 0,84 & 2,49 & 1,28 \\
\hline 12 & 0,83 & 0,88 & 0,58 & $-1,15$ & 1,08 & 3,01 & 0,85 \\
\hline 30 & 0,77 & 0,80 & 0,17 & $-1,98$ & 0,32 & 2,58 & 1,03 \\
\hline 52 & 1,03 & 1,04 & 0,02 & $-1,68$ & 0,14 & 1,54 & 0,91 \\
\hline 19 & 0,95 & 0,98 & $-0,05$ & $-1,83$ & $-0,05$ & 1,66 & 0,94 \\
\hline 03 & 1,03 & 1,04 & $-0,11$ & $-1,86$ & $-0,08$ & 1,36 & 0,85 \\
\hline 02 & 1,31 & 1,37 & $-0,57$ & $-2,10$ & $-0,78$ & 0,56 & 0,56 \\
\hline Média & 1,02 & 1,03 & 0,74 & - & - & - & - \\
\hline Desvio-padrão & 0,17 & 0,19 & 0,56 & - & - & - & - \\
\hline Fidedignidade dos itens & 0,99 & & & & & & \\
\hline Fidedignidade das pessoas & 0,80 & & & & & & \\
\hline
\end{tabular}

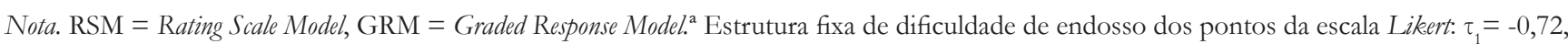
$\tau_{2}=0,00$ e $\tau_{3}=0,72$. Os parâmetros $\delta_{i}$ indicam a localização dessa estrutura para cada item; somar o valor $\delta_{i}$ aos parâmetros $\tau_{1}$, $\tau_{2}$ e $\tau_{3}$ resulta na dificuldade de endosso específica das categorias do item, analogamente aos parâmetros b do GRM.

e carga mínima nos demais fatores. Uma maneira de entender esses resultados é endossando uma visão realista da personalidade (Krueger, 2002). Nesse sentido, os fatores do instrumento não são meros rótulos para resumir comportamentos, mas sim representações matemáticas de influências biológicas reais subjacentes aos comportamentos.

De fato, a estrutura fatorial encontrada para o instrumento é coerente com modelos teóricos proeminentes de psicopatia com uma forte base biológica. Sellbom e Phillips (2012) conduziram uma análise fatorial exploratória de 17 escalas e subescalas avaliativas de psicopatia. Os autores encontraram três fatores representativos dos domínios fenotípicos do Modelo Triárquico da Psicopatia (Patrick et al., 2009): Meanness, explicando aspectos de insensibilidade, manipulação e egocentrismo; Disinhibition, explicando aspectos de impulsividade e comportamentos antissociais; e Boldness, explicando aspectos de dominância social, resiliência e ausência de medo. Dada a similaridade com os resultados do presente estudo, pode-se estabelecer uma correspondência entre os fatores do instrumento desenvolvido e o Modelo Triárquico da Psicopatia: Egocentrismo Maquiavélico proporciona 
Tabela 3

Análises de Teoria de Resposta ao Item da Escala Egocentrismo Maquiavélico (26 itens)

\begin{tabular}{|c|c|c|c|c|c|c|c|}
\hline \multirow{2}{*}{ Item } & \multicolumn{3}{|c|}{$\mathrm{RSM}^{\mathrm{a}}$} & \multicolumn{4}{|c|}{ GRM } \\
\hline & Infit & Outfit & $\delta_{i}$ & $b_{1}$ & $b_{2}$ & $b_{3}$ & $a$ \\
\hline 44 & 1,08 & 1,21 & 3,05 & 2,37 & 5,94 & 5,88 & 0,92 \\
\hline 54 & 1,34 & 0,99 & 2,85 & 3,08 & 5,02 & 5,67 & 1,77 \\
\hline 46 & 1,42 & 1,48 & 2,76 & 2,69 & 4,15 & 5,11 & 1,38 \\
\hline 37 & 1,42 & 1,51 & 2,60 & 2,18 & 3,92 & 4,44 & 1,06 \\
\hline 28 & 1,27 & 1,31 & 2,22 & 1,55 & 3,52 & 4,06 & 0,93 \\
\hline 45 & 1,21 & 1,25 & 2,06 & 1,24 & 3,01 & 4,34 & 0,80 \\
\hline 34 & 0,97 & 0,88 & 2,03 & 1,47 & 3,69 & 5,04 & 1,52 \\
\hline 04 & 1,22 & 1,55 & 1,80 & 0,76 & 2,82 & 3,68 & 0,40 \\
\hline 53 & 1,00 & 1,10 & 1,80 & 0,94 & 3,48 & 4,43 & 1,24 \\
\hline 31 & 0,99 & 0,90 & 1,78 & 1,01 & 2,89 & 4,96 & 1,31 \\
\hline 48 & 0,96 & 0,86 & 1,74 & 1,24 & 3,17 & 5,06 & 1,72 \\
\hline 20 & 1,16 & 1,31 & 1,72 & 0,66 & 2,67 & 3,87 & 0,55 \\
\hline 22 & 1,11 & 1,01 & 1,70 & 1,05 & 2,71 & 3,95 & 1,24 \\
\hline 36 & 0,85 & 0,86 & 1,70 & 0,90 & 3,22 & 5,14 & 1,57 \\
\hline 57 & 0,90 & 1,03 & 1,68 & 0,59 & 3,12 & 4,54 & 1,02 \\
\hline 05 & 1,04 & 1,11 & 1,64 & 0,63 & 2,63 & 4,22 & 0,87 \\
\hline 23 & 1,08 & 1,06 & 1,60 & 0,64 & 2,38 & 4,17 & 0,88 \\
\hline 27 & 0,88 & 1,02 & 1,53 & 0,20 & 3,09 & 4,21 & 0,66 \\
\hline 13 & 1,40 & 1,48 & 1,46 & 0,79 & 1,93 & 3,00 & 0,86 \\
\hline 21 & 0,97 & 0,92 & 1,44 & 0,49 & 2,40 & 4,11 & 1,23 \\
\hline 16 & 0,90 & 0,85 & 1,40 & 0,76 & 2,47 & 4,75 & 1,81 \\
\hline 14 & 0,90 & 0,83 & 1,36 & 0,45 & 2,35 & 4,32 & 1,46 \\
\hline 56 & 0,82 & 0,76 & 1,33 & 0,36 & 2,49 & 4,58 & 1,64 \\
\hline 17 & 0,93 & 0,84 & 1,31 & 0,50 & 2,24 & 4,36 & 1,64 \\
\hline 55 & 0,99 & 1,00 & 0,50 & $-0,73$ & 0,71 & 2,57 & 1,25 \\
\hline 29 & 0,98 & 1,10 & 0,04 & $-1,93$ & $-0,08$ & 2,50 & 0,49 \\
\hline Média & 1,07 & 1,09 & 1,73 & - & - & - & - \\
\hline Desvio-padrão & 0,18 & 0,23 & 0,63 & - & - & - & - \\
\hline Fidedignidade dos itens & 0,99 & & & & & & \\
\hline Fidedignidade das pessoas & 0,75 & & & & & & \\
\hline
\end{tabular}

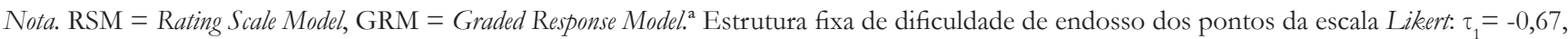
$\tau_{2}=0,01$ e $\tau_{3}=0,66$. Os parâmetros $\delta_{i}$ indicam a localização dessa estrutura para cada item; somar o valor $\delta_{i}$ aos parâmetros $\tau_{1}$, $\tau_{2}$ e $\tau_{3}$ resulta na dificuldade de endosso específica das categorias do item, analogamente aos parâmetros b do GRM.

informação sobre o domínio Meanness, Descontrole é mais avaliativo de Disinbibition, e Audácia avalia características de Boldness.

A correspondência entre os fatores do instrumento e os domínios fenotípicos do Modelo Triárquico também recebe apoio das análises correlacionais, cujos resultados são coerentes com as expectativas teóricas.
O fator Descontrole, assim como Disinhibition no estudo de Sellbom e Phillips (2012), esteve mais fortemente relacionado ao BAS-fun seeking $(0,50)$; Audácia, assim como Boldness, esteve mais relacionado ao BIS $(-0,53)$. A única exceção foi Egocentrismo Maquiavélico que, em vez de mais fortemente relacionado ao BIS (-0,12 no presente estudo, e $-0,34$ no estudo de 
Tabela 4

Análises de Teoria de Resposta ao Item da Escala Audácia (13 itens)

\begin{tabular}{lccccccc}
\hline \multirow{2}{*}{ Item } & \multicolumn{3}{c}{ RSM $^{\mathrm{a}}$} & \multicolumn{5}{c}{ GRM } \\
\cline { 2 - 8 } & Infit & Onffit & $\delta_{i}$ & $b_{1}$ & $b_{2}$ & $b_{3}$ & $a$ \\
\hline 60 & 1,26 & 1,06 & 2,37 & 2,03 & 4,13 & 4,49 & 1,04 \\
49 & 0,82 & 0,74 & 1,41 & 0,54 & 3,00 & 3,93 & 1,16 \\
58 & 1,18 & 1,25 & 1,39 & 0,63 & 2,28 & 3,51 & 0,70 \\
50 & 1,10 & 0,98 & 1,09 & 0,74 & 2,44 & 3,60 & 2,09 \\
59 & 0,98 & 0,97 & 1,00 & 0,31 & 2,20 & 3,54 & 1,80 \\
39 & 0,99 & 1,02 & 0,98 & $-0,06$ & 1,73 & 3,04 & 0,77 \\
25 & 1,24 & 1,22 & 0,83 & 0,01 & 1,22 & 2,33 & 0,61 \\
51 & 0,86 & 0,88 & 0,38 & $-1,12$ & 0,64 & 2,50 & 0,69 \\
32 & 0,99 & 1,06 & 0,31 & $-1,07$ & 0,45 & 2,28 & 0,55 \\
33 & 1,08 & 1,09 & $-0,09$ & $-1,43$ & $-0,12$ & 1,15 & 0,83 \\
40 & 0,94 & 0,97 & $-0,17$ & $-1,82$ & $-0,32$ & 1,35 & 0,61 \\
8 & 1,18 & 1,19 & $-0,38$ & $-1,64$ & $-0,55$ & 0,58 & 0,60 \\
9 & 0,85 & 0,86 & $-0,47$ & $-2,36$ & $-0,89$ & 0,94 & 0,87 \\
Média & 1,04 & 1,02 & 0,67 & - & - & - & - \\
Desvio-padrão & 0,14 & 0,14 & 0,80 & - & - & - & - \\
Fidedignidade dos itens & 1,00 & & & & & & \\
Fidedignidade das pessoas & 0,64 & & & & & & - \\
\hline
\end{tabular}

Nota. RSM = Rating Scale Model, GRM = Graded Response Model. ${ }^{2}$ Estrutura fixa de dificuldade de endosso dos pontos da escala Likert. $\tau_{1}=-0,51$, $\tau_{2}=0,02$ e $\tau_{3}=0,50$. Os parâmetros $\delta_{i}$ indicam a localização dessa estrutura para cada item; somar o valor $\delta_{i}$ aos parâmetros $\tau_{1}$, $\tau_{2}$ e $\tau_{3}$ resulta na dificuldade de endosso específica das categorias do item, analogamente aos parâmetros b do GRM.

Sellbom \& Phillips, 2012), correlacionou-se com BAS-drive $(0,19)$ e BAS-fun seeking $(0,22)$. Ou seja, o fator Egocentrismo Maquiavélico resultou mais saturado em aspectos de internalização e externalização do que a dimensão Meanness. É possível, portanto, que tenha resultado contendo aspectos mais severos de psicopatologia (por exemplo, hostilidade e desprezo) do que outros instrumentos de autorrelato de psicopatia. A curva de informação do fator Egocentrismo Maquiavélico sustenta essa hipótese, mostrando que os itens funcionaram melhor na porção extrema superior do traço latente. Em vez de uma limitação, isso garante ao instrumento um potencial uso em estudos com amostras forenses.

Os fatores, também, podem ser entendidos a partir de um modelo dual de psicopatia. Fowles e Dindo (2006) reuniram evidências que mostram ser a psicopatia o resultado de duas influências neurobiológicas distintas: déficits na aprendizagem aversiva e baixo controle dos impulsos. Mais popularmente, essa dicotomia aparece na literatura como psicopatia primária (afetos positivos, falta de empatia e frieza emocional) e secundária (afetos negativos, impulsividade e falta de planejamento; ver Lykken, 1995). É possível mapear os fatores Egocentrismo Maquiavélico e Descontrole também quanto a essas duas dimensões. Embora ambos os fatores tenham se relacionado a altos níveis de afetos negativos e baixos níveis de afetos positivos, Egocentrismo Maquiavélico se mostrou mais fortemente relacionado à psicopatia primária da escala $\operatorname{LSRP}(0,66)$ do que à psicopatia secundária (0,36); Descontrole se mostrou mais relacionado à psicopatia secundária $(0,61)$ do que aos aspectos primários $(0,46)$, também explicando o envolvimento prévio com a lei, coerente com a teoria sobre a psicopatia secundária (Lykken, 1995). Além disso, a porção mais positiva da psicopatia primária parece ter sido mais bem capturada pelo fator Audácia, que apresentou correlação positiva com afetos positivos $(0,23)$ e negativa com afetos negativos $(-0,42)$. Isso pode explicar a quase ortogonalidade observada entre Audácia e os demais fatores.

O fator Audácia mostrou correlatos análogos aos de uma das principais ferramentas para avaliar Boldness - a escala Fearless Dominance (FD)do instrumento 

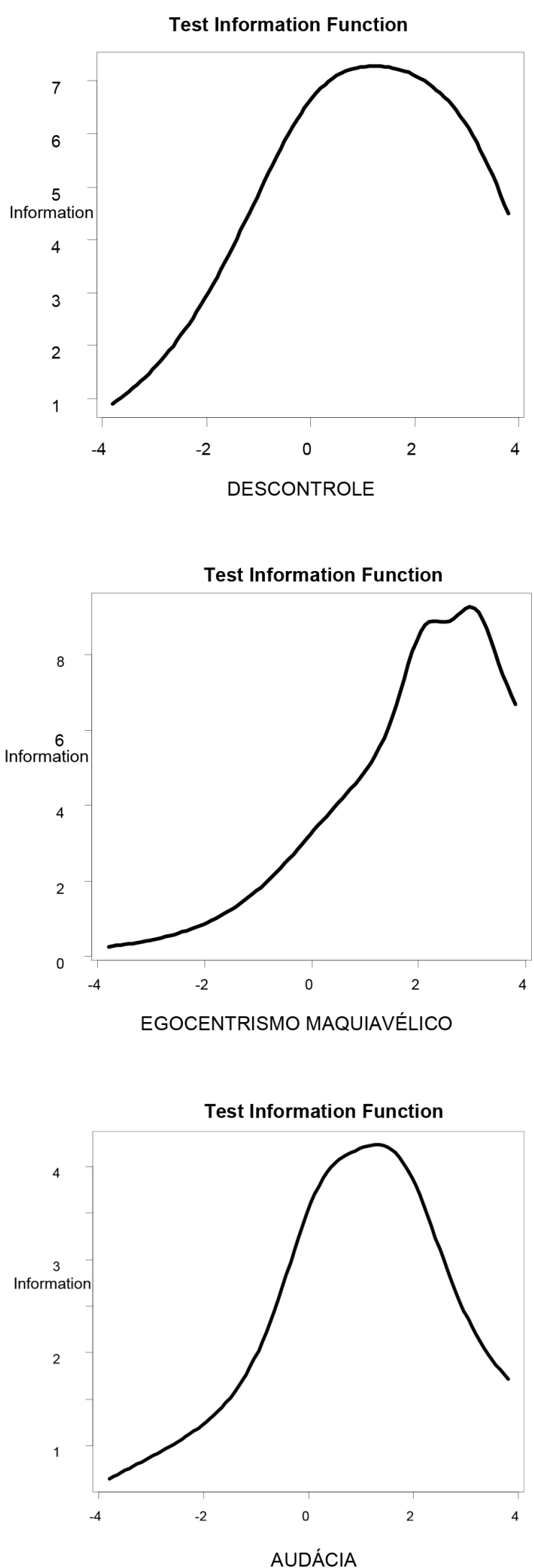

Figura 1. Curvas de informação para as escalas
PPI (Lilienfeld \& Andrews, 1996). Na metanálise de Miller e Lynam (2012), FD obteve correlações de nulas a pequenas com outros instrumentos de psicopatia; na metanálise de Marcus, Fulton e Edens (2013), as correlações foram de nulas a moderadas. Esse padrão reflete o fato de que Boldness tende a ser fracamente correlacionado aos demais domínios fenotípicos (ver também Sellbom \& Phillips, 2012). De fato, há uma discussão ainda em andamento na literatura sobre se aspectos positivos como Boldnesse FD devem ser considerados parte constituinte da psicopatia (Lynam \& Miller, 2012; Marcus et al., 2013; Miller \& Lynam, 2012). Não obstante, há evidências de que a manipulação, a frieza emocional e a impulsividade, somadas à baixa ansiedade e ao reduzido medo (Boldness), resultam em uma configuração de déficits atencionais e cognitivos que intensificam ainda mais os prejuízos no processamento emocional (Corr, 2010). Assim, embora enfocando em aspectos que contrastam com os fatores Egocentrismo Maquiavélico e Descontrole, o fator Audácia proporciona informação útil para futuros estudos na área.

Secundariamente, o presente estudo também representa uma contribuição no sentido de desmistificar a crença de que não é possível avaliar traços de psicopatia usando o autorrelato. Embora a psicopatia acarrete distorções e vieses de resposta em situações de avaliação que suscitam, tipicamente, alta desejabilidade social (como seleções para empregos e concursos; Edens, 2004), o mesmo não se generaliza a contextos de pesquisa. Em uma metanálise de 45 estudos que se utilizaram dos mais variados instrumentos de avaliação, Ray et al. (2013) encontraram correlações quase nulas entre psicopatia e tendência a respostas desejáveis $(r=-0,11$ até $-0,16)$ e correlações positivas - embora pequenas entre psicopatia e tendências a exacerbar psicopatologia externalizante $(r=0,27$ até 0,32$)$. Em outras palavras, a literatura empírica falseia o mito de que traços de psicopatia levam, necessariamente, a vieses ao responder a instrumentos de autorrelato. Instrumentos de autorrelato são amplamente utilizados para a pesquisa em muitos contextos culturais e representam uma maneira econômica e vantajosa de avaliação de traços de psicopatia (Lilienfeld \& Fowler, 2006).

É importante ressaltar que o presente instrumento não se destina à avaliação de psicopatia em contextos de seleção profissional, exame pericial ou qualquer situação em que haja incentivos explícitos para falsear respostas. Não obstante, trata-se de uma ferramenta adequada para uso em situações de pesquisa, da mesma forma como usualmente feito com instrumentos de 
Tabela 5

Correlações Bivariadas

\begin{tabular}{lllllllllll}
\hline & BAS & BAS-RR & BAS-D & BAS-FS & BIS & AP & AN & LSRP-PP & LSRP-PS & LSRP-total \\
\hline Descontrole & $0,38^{* *}$ & 0,07 & $0,26^{* *}$ & $0,50^{* *}$ & $-0,08^{* *}$ & $-0,26^{* *}$ & $0,42^{* *}$ & $0,46^{* *}$ & $0,61^{* *}$ & $0,62^{* *}$ \\
Egocentrismo & $0,18^{* *}$ & $-0,04$ & $0,19^{* *}$ & $0,22^{* *}$ & $-0,12^{* *}$ & $-0,23^{* *}$ & $0,26^{* *}$ & $0,66^{* *}$ & $0,36^{* *}$ & $0,65^{* *}$ \\
$\begin{array}{l}\text { Maquiavélico } \\
\text { Audácia }\end{array}$ & $-0,13^{* *}$ & $-0,20^{* *}$ & $-0,05$ & $-0,06$ & $-0,53^{* *}$ & $0,23^{* *}$ & $-0,42^{* *}$ & $-0,03$ & $-0,22^{* *}$ & $-0,12^{* *}$ \\
\hline
\end{tabular}

Nota. BAS = Behavioral Activation, BAS-D = Behavioral Activation - Reward Responsiveness, BAS-D = Behavioral Activation - Drive, BAS-FS $=$ Behavioral Activation - Fun Seeking, BIS = Behavioral Inbibition System, AP = afetos positivos, AN = afetos negativos, LSRP-PP = Levenson Self-Report Psychopathy - Psicopatia Primária, LSRP-PS = Levenson Self-Report Psychopatby - Psicopatia Secundária, LSRP-total $=$ Levenson Self-Report Psychopathy - escore total.

$* p<0,05 * * p<0,01$

autorrelato que avaliam outros aspectos da personalidade e da psicopatologia.

\section{Considerações Finais}

O instrumento construído no presente estudo se mostrou adequadamente avaliativo dos fatores Egocentrismo Maquiavélico, Audácia e Descontrole, análogos aos domínios do Modelo Triárquico da Psicopatia (Patrick et al., 2009). Limitações do estudo dizem respeito à amostra de conveniência empregada, que torna necessárias, ainda, outras investigações sobre as propriedades psicométricas dos instrumentos. Por exemplo, a invariância dos parâmetros ao longo de diferentes grupos populacionais deve ser explorada em futuros estudos sobre funcionamento diferencial. Ressalta-se, também, o fato de o envolvimento com a lei ter sido avaliado por meio do autorrelato e de questão única dicotômica, quando seria mais informativo ter acesso a um registro formal de cada participante - sugestão para novas investigações com um caráter mais clínico e controlado.

Em função da natureza correlacional deste trabalho, não foi possível testar em que medida os fatores proporcionam informação sobre alguns dos aspectos neurobiológicos que motivaram a construção do instrumento (por exemplo, startle). Logo, seria útil investigar correlações com marcadores fisiológicos e neuropsicológicos da psicopatia. Outra necessidade crucial é verificar a validade discriminante do instrumento o que diz respeito a identificar grupos de prisioneiros com altos escores de acordo com a escala PCL-R. Trata-se de uma investigação psicométrica necessária, que ajudará a justificar a afirmação de que o instrumento é informativo das mesmas características capturadas pela principal ferramenta de avaliação clínica e forense da área.

\section{Referências}

Andrich, D. (1978). A rating formulation for ordered response categories. Psychometrika, 43(4), 561-573. doi:10.1007/BF02293814

Brinkley, C. A., Schmitt, W. A., Smith, S. S., \& Newman, J. P. (2001). Construct validation of a self-report psychopathy scale: Does Levenson's self-report psychopathy scale measure the same constructs as Hare's psychopathy checklist-revised? Personality and Individual Differences, 31(7), 1021-1038. doi:10.1016/S0191-8869(00)00178-1

Brock, R. L., Barry, R. A., Lawrence, E., Dey, J., \& Rolffs, J. (2012). Internet administration of paper-and-pencil questionnaires used in couple research: assessing psychometric equivalence. Assessment, 19(2), 226-242. doi:10.1177/1073191110382850

Carver, C. S., \& White, T. L. (1994). Behavioral inhibition, behavioral activation, and affective responses to impending reward and punishment: The BIS/ BAS Scales. Journal of Personality and Social Psychology, 67(2), 319-333. doi:10.1037/0022-3514.67.2.319

Cleckley, H. (1976). The mask of sanity (5th ed.). St. Louis, MO: Mosby.

Cooke, D. J., Kosson, D. S., \& Michie, C. (2001). Psychopathy and ethnicity: Structural, item, and test generalizability of the Psychopathy Checklist-Revised (PCL-R) in Caucasian and African American Participants. Psychological Assessment, 13(4), 531-542. 
Cooke, D. J., \& Michie, C. (2001). Refining the construct of psychopathy: Towards a hierarchical model. Psychological Assessment, 13(2), 171-188.

Corr, P. J. (2010). The psychoticism-psychopathy continuum: A neuropsychological model of core deficits. Personality and Individual Differences, 48(6), 695-703. doi:10.1016/j.paid.2009.12.023

Corr, P. J., \& McNaughton, N. (2008). Reinforcement sensitivity theory and personality. In P. J. Corr (Ed.), The Reinforcement Sensitivity Theory of Personality (pp. 115-187). New York: Cambridge University Press.

Davoglio, T. R., Gauer, G. J. C., Vasconcellos, S. J. L., \& Lühring, G. (2011). Medida Interpessoal de Psicopatia (IM-P): Estudo preliminar no contexto brasileiro. Trends in Psychiatry and Psychotherapy, 33(3), 147-155. doi:10.1590/S2237-60892011000300004

Dindo, L., \& Fowles, D. (2011). Dual temperamental risk factors for psychopathic personality: Evidence from self-report and skin conductance. Journal of Personality and Social Psychology, 100(3), 557-566. doi: $10.1037 / \mathrm{a} 0021848$

Edens, J. F. (2004). Effect of response distortion on the assessment of divergent facets of psychopathy. Assessment, 11(1), 109-12.

Edens, J. F., Poythress, N. G., Lilienfeld, S. O., Patrick, C. J., \& Test, A. (2008). Further evidence of the divergent correlates of the Psychopathic Personality Inventory factors: Prediction of institutional misconductamongmale prisoners. PsychologicalAssessment, 20(1), 86-91. doi:10.1037/1040-3590.20.1.86

Embretson, S. E., \& Reise, S. P. (2000). Item response theory forpsychologists. Mahwah, New Jersey: Lawrence Earlbaum Associates, Publishers.

Flor, H., Birbaumer, N., Hermann, C., Ziegler, S., \& Patrick, C. J. (2002). Aversive Pavlovian conditioning in psychopaths: peripheral and central correlates. Psychophysiology, 39(4), 505-18. doi:10.1017. S0048577202394046

Forth, A. E., Kosson, D. S., \& Hare, R. D. (1995). Hare psychopathy checklist: Youth version. Toronto: Multi-Health Systems.

Fowles, D. C., \& Dindo, L. (2006). A dual-deficit model of psychopathy. In C. J. Patrick (Ed.), Handbook of Psychopathy (pp. 14-34). New York: The Guilford Press.
Hare, R. D. (2003). Manual for the revised psychopatby checklist $\left(2^{a}\right.$ edition). Toronto, Canadá: Multi-Health Systems.

Hauck-Filho, N., \& Teixeira, M. A. P. (2014). Revisiting the psychometric properties of the Levenson self-report psychopathy scale. Journal of Personality Assessment, 96(4), 459-64. doi:10.1080/00223891.2 013.865196

Hughes, K. A., Moore, R. A., Morris, P. H., \& Corr, P. J. (2012). Throwing light on the dark side of personality: Reinforcement sensitivity theory and primary/secondary psychopathy in a student population. Personality and Individual Differences, 52(4), 532-536. doi:10.1016/j.paid.2011.11.010

Kosson, D. S., Steuerwald, B. L., Forth, A. E., \& Kirkhart, K. J. (1997). A new method for assessing the interpersonal behavior of psychopathic individuals: Preliminary validation studies. Psychological Assessment, 9(2), 89-101. doi:10.1037/1040-3590.9.2.89

Krueger, R. F. (2002). Personality from a realist's perspective: Personality traits, criminal behaviors, and the externalizing spectrum. Journal of $R e-$ search in Personality, 36(6), 564-572. doi:10.1016/ S0092-6566(02)00506-8

Levenson, M. R., Kiehl, K. A., \& Fitzpatrick, C. M. (1995). Assessing psychopathic attributes in a noninstitutionalized population. Journal of Personality and Social Psychology, 68(1), 151-158.

Lilienfeld, S., \& Fowler, K. A. (2006). The self-report assessment of psychopathy: Problems, pitfalls, and promises. In C. J. Patrick (Ed.), Handbook of Psychopathy (pp. 107-132). New York: The Guilford Press.

Lilienfeld, S. O., \& Andrews, B. P. (1996). Development and preliminary validation of a self-report measure of psychopathic personality traits in noncriminal populations. Journal of Personality Assessment, 66(3), 488-524. doi:10.1207/s15327752jpa6603_3

Linacre, J. M. (2011). A user's guide to Winsteps, Program Manual 3.74.0. Chicago: Winsteps.com.

Lykken, D. T. (1995). The antisocial personalities (1 ${ }^{\mathrm{a}} \mathrm{ed}$.). Hillsdale, New Jersey: Lawrence Earlbaum Associates, Inc.

Lynam, D. R., \& Miller, J. D. (2012). Fearless dominance and psychopathy: A response to Lilienfeld et al. Personality Disorders, 3(3), 341-53. doi:10.1037/ a0028296 
Marcus, D. K., Fulton, J. J., \& Edens, J. F. (2013). The two-factor model of psychopathic personality: Evidence from the psychopathic personality inventory. Personality Disorders, 4(1), 67-76.

Miller, J. D., \& Lynam, D. R. (2012). An examination of the psychopathic personality inventory's nomological network: a meta-analytic review. Personality Disorders, 3(3), 305-26. doi:10.1037/a0024567

Morana, H. (2004). Escala hare PCL-R: Critérios para pontuação de psicopatia revisados. Versão brasileira. São Paulo, SP: Casa do Psicólogo.

Muthén, B., du Toit, S. H. C., \& Spisic, D. (1997). Robust inference using weighted least squares and quadratic estimating equations in latent variable modeling with categorical and continuous outcomes. Non-Published Technical Report.

Newman, J. P., \& Malterer, M. B. (2009). Problems with the BIS/BAS scales or Lykken's model of primary psychopathy? A reply to. Personality and iIdividual dDfferences, 46(7), 673-677. doi:10.1016/j. paid.2009.01.037

Patrick, C. J., Fowles, D. C., \& Krueger, R. F. (2009). Triarchic conceptualization of psychopathy: developmental origins of disinhibition, boldness, and meanness. Development and Psychopathology, 21(3), 913-938. doi:10.1017/S0954579409000492

Ray, J. V, Hall, J., Rivera-Hudson, N., Poythress, N. G., Lilienfeld, S. O., \& Morano, M. (2013). The relation between self-reported psychopathic traits and distorted response styles: a meta-analytic review. Personality Disorders, 4(1), 1-14. doi:10.1037/ a0026482

Rizopoulos, D. (2006). ltm: An R package for latent variable modelling and item response theory analyses. Journal of Statistical Software, 17(5), 1-25.
Ronchetti, R., Davoglio, T. R., Salvador-Silva, R., Vasconcellos, S. J. L., \& Gauer, G. J. C. (2010). Inventário de psicopatia de Hare versão jovens (PCL:YV): Estudo preliminar em amostra adolescente brasileira. Interamerican Journal of Psychology, 44(3), 540-546.

Samejima, F. (1969). Estimation of latent trait ability using a response pattern of graded scores. Psychometrika Monograph Supplement No. 17, 34(4, Pt. 2).

Sellbom, M., \& Phillips, T. R. (2013). An examination of the triarchic conceptualization of psychopathy in incarcerated and nonincarcerated samples. Journal of Abnormal Psychology, 122(1), 208-214. doi:10.1037/a0029306

Skeem, J. L., Polaschek, D. L. L., Patrick, C. J., \& Lilienfeld, S. O. (2011). Psychopathic personality bridging the gap between scientific evidence and public policy. Psychological Science in the Public Interest, 12(3), 95-162. doi:10.1177/1529100611426706

Velicer, W. (1976). Determining the number of components from the matrix of partial correlations. Psychometrika, 41(3), 321-327. doi:10.1007/ BF02293557

Wilson, K., Juodis, M., \& Porter, S. (2011). Fear and loathing in psychopaths: A meta-analyticinvestigation of the facial affect recognition deficit. Criminal Justice and Behavior, 38(7), 659-668. doi:10.1177/0093854811404120

Zanon, C., Bastianello, M. R., Pacico, J. C., \& Hutz, C. S. (2013). Desenvolvimento e validação de uma escala de afetos positivos e negativos. Psico-USF, 18(2), 193-201. doi:10.1590/S1413-82712013000200003

Recebido: 05/11/2013

$1^{a}$ reformulação: 01/09/2014

Aprovado: 22/12/2014 
Nota dos autores:

O presente estudo recebeu financiamento da Coordenação de Aperfeiçoamento de Pessoal de Nível Superior (CAPES), na forma de bolsa de doutorado ao primeiro autor.

Nota sobre os autores

Sobre os autores:

Nelson Hauck Filho é pós-doutorando em Psicologia pela Universidade São Francisco - Itatiba.

Roberta Salvador-Silva é mestranda em Psicologia pela Pontifícia Universidade Católica do Rio Grande do Sul.

Marco Antônio Pereira Teixeira é professor adjunto do Programa de Pós-Graduação em Psicologia, pela Universidade Federal do Rio Grande do Sul.

Contato com os autores:

Universidade Federal do Rio Grande do Sul, Brasil

Rua Ramiro Barcellos, 2600, sala 117

Porto Alegre, Rio Grande do Sul, Brasil

CEP: 90035-003

E-mail: hauck.nf@gmail.com 